\title{
A novel bio-inspired routing algorithm based on ACO for WSNs
}

\author{
Afsah Sharmin, F. Anwar, S. M. A. Motakabber \\ Department of Electrical and Computer Engineering, International Islamic University Malaysia, \\ Kuala Lumpur, Malaysia
}

\begin{abstract}
Article Info
Article history:

Received Jan 14, 2019

Revised Mar 13, 2019

Accepted Mar 30, 2019

\section{Keywords:}

$\mathrm{ABC}$

ACO algorithm

IoT

Network

PSO

Routing

WSN

ABSTRACT

The methods to achieve efficient routing in energy constrained wireless sensor networks (WSNs) is a fundamental issue in networking research. A novel approach of ant colony optimization (ACO) algorithm for discovering the optimum route for information transmission in the WSNs is proposed here for optimization and enhancement. The issue of path selection to reach the nodes and vital correspondence parameters, for example, the versatility of nodes, their constrained vitality, the node residual energy and route length are considered since the communications parameters and imperatives must be taken into account by the imperative systems that mediate in the correspondence procedure, and the focal points of the subterranean insect framework have been utilized furthermore. Utilizing the novel technique and considering both the node mobility and the existing energy of the nodes, an optimal route and best cost from the originating node to the target node can be detected. The proposed algorithm has been simulated and verified using MATLAB and the simulation results demonstrate that new ACO based algorithm achieved improved performance, about 30\% improvement compared with the traditional ACO algorithm, and faster convergence to determine the best cost route, and recorded an improvement in the energy consumption of the nodes per transmission.
\end{abstract}

Copyright $@ 2019$ Institute of Advanced Engineering and Science. All rights reserved.

\section{Corresponding Author:}

F. Anwar,

Department of Electrical and Computer Engineering,

International Islamic University Malaysia,

Jalan Gombak, 53100 Kuala Lumpur, Malaysia.

Email: farhat@iium.edu.my

\section{INTRODUCTION}

The WSN is a significant accumulation of autonomous dynamic sensor nodes to screen certain physical conditions working in a fundamental zone and systematized into accommodating the network. Every node comprises of at least one or more sensors, memory, a battery which is rechargeable/non-rechargeable, and a processing unit with certain capabilities. Besides WSN hubs are controlled by battery which has constrained vitality and is troublesome to be revived. The lifetime of the sensor hub depends to a huge degree on the battery life, and the outlandish energy utilization will cause the system to pass on impetuously and lessen the system lifetime. So how to plan the WSN route calculation algorithm which can spare node vitality and enhance the nature of system correspondence is the key point for WSN. The information transmission is performed in multiple hop strategy in WSN and the gathered information is exchanged with the user via the sink [1]. A sink is the entity where information is required and the sink might be available far away from the data bearing hub. Once installed in the genuine domain, the sensor node connects to different nodes and the sensor networks autonomously transfer information utilizing diverse routing protocols back to the sink node. If we can solve the related research issues, the WSN has incredible, long span potential for changing our 
everyday lives [2]. Routing is a dynamic research focus in WSNs with numerous promising methods and coordinating with novel strategies and setting up another exploration field are future patterns [3].

The routing protocols ought to be profoundly proficient to encourage the successful transmission of information of nodes among each other due to asset limitations and ecological obstruction. The suitability of a particular routing convention primarily relies upon the proficiencies of the nodes and on the application requirements [4]. To confront changed difficulties in impenetrable and complex conditions, for example, military, generation, restorative and numerous different situations, conventional or traditional routing protocols of WSNs are not vitality proficient as well as adaptable. For performing effectual routing and keeping up energy effectiveness among nodes amid information transmission, the bio-roused procedures are actualized in WSN called as Swarm Intelligence which shows as a gathering of self-regulating species [5]. Usually well-known Swarm Intelligence strategies are ACO, Particle Swarm Optimization and Artificial Bee Colony [6]. Calculations in view of Swarm Intelligence, nature-based knowledge is profoundly strong, versatile and adaptable. ACO is a self-motivated and decentralized system for approximate optimization which is reasonable to take care of different combinatorial issues and stochastic issue in WSNs. The primary aim of appropriateness of ACO in WSNs is the comparative attributes of both where ACO can essentially perform in unique topology, neighbourhood tasks, hubs with bounds, multiway and connection excellence [7]. ACO is motivated from the conduct of genuine ants searching for nourishment and this attribute of ant colonies is exploited in ACO algorithm [8]. ACO is a meta-heuristic method and can solve for instance discrete optimization problems. The choice of nodes depends on the probability equation and further the pheromone trail reinstate is additionally in view of the pheromone revised formula [9].

In [10], a noble and unique routing algorithm, called EICAntS, efficient IoT communications based on ant system, is proposed to enhance the path determination system by utilizing the benefits of an insect province framework, ant colony based method. The energy perspective is considered and the global effectiveness factor is figured which relates to the pheromone measurements in the ant insect framework. The assessment results of EICAntS recorded an enhancement in average end to end delay, throughput, node lifetime and preservation of vitality. The proposed calculation treats only the instance of Internet of Things, not considering with regards to WSN. No facts were given to the most proficient method to evaluate the mobility. The usage of the energy parameter by the proposed calculation requires additionally demonstrating by considering the diverse components impacting energy or vitality utilization. The energy impact only considers the data or information class treated by the node.

An enhanced ACO for a critical node or less energy node for data or information transmission is proposed in [11]. In case of no other path is there, the ill or low energy node just exchange information where the information is transmitted based on priority or need. The rehashed demand of a similar packet ID prompts higher need which promotes further information or data exchange. The transmission of data can be hindered because of the low energy node. The less energy nodes only forward the packets without processing the data. The critical nodes essentially unicast the information to next neighbor nodes while not needing to wait for an acknowledgment.

An amended ant colony optimization based approach for optimal route path discovery in WSNs is presented in [12]. An optimum route detection algorithm is proposed here and the optimum path is detected by intelligent ants those have some data with respect to nodes in the path for routing. No specifics were given by the proposed procedure of the most proficient method to measure the energy parameter.

An ant-based route calculation method to locate the best routes between a nourishment source and their nests is proposed in [13]. Here, for optimization the concentration of pheromone is influenced by the combination of the pheromone and the node energy and node delay. It permits dynamically modifying the parameters amid the quest for the route. The pheromone refresh rules with reward and discipline component are embraced. The energy efficiency parameter is considered by the calculation, to guarantee energy proficient utilization and afterward to prolong the life cycle of the network system, requiring to consider the distinctive factors affecting energy utilization. No details were given by the proposed algorithm on how to estimate the delay factor.

In [14], a TA-NARP algorithm is proposed which is the improvement in novel routing protocol NARP in light of ACO for expanding throughput. By executing PA-SHORT calculation with NARP, the upgrade is done where PA-SHORT finds the most diminutive cut route with the best-discovered paths that decreases the number of hops.

$\mathrm{T}$ Camilo et al. present the IABR, improved ant-based routing, and EEABR, energy efficient ant based routing, calculations and algorithms [15]. They construct a unique function to ascertain pheromone left by the backward ants on their path to the nest, however the algorithm does not reflect energy stability of the whole network system. This is critical to the WSN. The EEABR convention was structured with a target to improve sensor nodes vitality by decreasing correspondence overhead in finding the routes from source to 
destination. The protocol includes new functionalities in pheromone tables, refreshing of sensor hubs. The other downside of EEABR is absence of QoS and to some degree delay in packet conveyance.

This paper presents a noble algorithm based on an ant colony algorithm which considers the node mobility and both the node energy and the route path. The majority of the proposed routing solutions or algorithms have tended to only some correspondence parameters for communication in the network when taking care of the routing issue. The whole particular features, highlights and parameters are not considered for effectual communications inside WSNs. Some works have considered the vitality factor, yet its misuse is not ideal since they do not take into account the distinctive components affecting this parameter. Settling the issue of routing by thinking about a few parameters is not adequate to attain the correspondence quality desired by the energy constrained WSNs applications particularly in complex and dense environments. For this reason, here an efficient and fundamental routing calculation exploiting the advantages of ant based system for the improvement in communications in finding the optimum path for data transmission is proposed and the vital correspondence parameters, for example, versatility, vitality and route length, are considered [16].

\section{SYSTEM MODEL}

\subsection{The network model}

In order to make the routing algorithm more general, the network model assumes that the sensor nodes are haphazardly dispensed in a rectangular region. Also, the links are symmetrical. On the off chance that the objective's transmitted power is known, nodes can compute the approximate distance of the senders as per the Received Signal Strength Indication. In the meantime, the following presumptions are made for sensor nodes:

a. For all sensor nodes the preliminary energy is the same;

b. All nodes are similar in form or isomorphic;

c. Each and every node can get the data of its neighbor sensor nodes;

\subsection{Proposed ACO algorithm}

It is important to enhance network routing algorithm, keeping in mind the end goal to find the best cost route efficiently for network optimization and to decrease the system vitality utilization. At time t, each ant selects the following path for the proposed improvement of the ant colony optimization algorithm, as indicated by a following probabilistic formula:

$$
P_{i j}^{m}=\left\{\begin{array}{c}
\frac{\left[\tau_{i j}(t)\right]^{\alpha}\left[\eta_{i j}(t)\right]^{\beta}\left[\vartheta_{i j}(t)\right]^{\gamma} E_{j}}{\sum_{s \subset \text { allowed }_{m}\left[\tau_{i s}(t)\right]^{\alpha}\left[\eta_{i s}(t)\right]^{\beta}\left[\vartheta_{i s}(t)\right]^{\gamma} E_{s}}} \quad, j \subset \text { allowed }_{m} \\
0, \text { others }
\end{array}\right.
$$

here, $\eta_{i j}(t)=\frac{1}{d_{i j}}$

where $\tau_{i j}(t)$ is the pheromone intensity on edge $i, j$ and $\eta_{i j}(t)$ is the desirability of edge $i, j$ which is heuristic information on edge $(i, j)$, two impact factors $\alpha$ and $\beta$ that control the influence of pheromone intensity and heuristic information correspondingly. According to the average mobility, speed of the nodes, $\vartheta_{i j}(t)$ which is the stability factor is calculated where $\gamma$ is the constant. To increase or decrease the effect of the stability factor $\gamma$ is used. The residual energy of a node is $E_{j}$ that ant $m$ will visit. The distance between $i$ and $j$ is $d_{i j}$. According to the following equation the amount of pheromone is updated:

$$
\begin{aligned}
& \tau_{i j}(t+1)=(1-\rho) \tau_{i j}(t)+\Delta \tau_{i j}(t) \\
& \Delta \tau_{i j}(t)=\sum_{k=1}^{m} \Delta \tau_{i j}^{k}
\end{aligned}
$$

where $\rho$ is evaporation coefficient of pheromone, $\Delta \tau_{i j}(t)$ is the amount of pheromone increased or deposited of edge $i, j$, usually given by,

$$
\Delta \tau_{i j}^{k}=\left\{\begin{array}{lr}
\frac{Q}{L_{k}} \text { if ant } k \text { uses on edge }(i, j) \\
0 \quad \text { otherwise }
\end{array}\right.
$$


where pheromone strength is represented by the constant Q, $L_{k}$ is the length of the path of the $k^{\text {th }}$ ant's tour.

\subsection{Energy model}

The radio model is incorporated by the proposed method where [17] and [18] discussed the radio model. When the distance is less than a threshold value $d_{0}$ a free space model is used for energy consumption by the sensor nodes for information transmission. Else, the multi-path model is being used and the equation for energy consumption by the sensor nodes is given by:

$$
E_{\text {tran }}(l, d)=\left\{\begin{array}{r}
l E_{\text {elec }}+l \epsilon_{f s} d^{2} \quad \text { if } d<d_{0} \\
l E_{\text {elec }}+l \epsilon_{m p} d^{4} \quad \text { if } d \geq d_{0}
\end{array}\right.
$$

Where an $l$-bit data are transmitted over a distance $d$. Power dissipation is denoted here by $E_{\text {elec }}$. Energy consumed by the amplifier in free space is given by $\epsilon_{f s}$. And the energy consumed by the multi-path model is given by $\epsilon_{m p}$.

If $E_{\text {tot }}$ is the total initial level energy of a sensor node and $E_{t r a n}$ is the transmission energy then the residual energy level of a sensor node for instance $n_{i}$ is computed as follows:

$$
E_{\text {resi }}=E_{\text {tot }_{i}}-E_{\text {tran }_{i}}
$$

\subsection{Fitness function}

The existing node energy level is not considered for searching the lowest cost route in the conventional ACO technique. It might prompt sudden passing of a few nodes, and afterward influence the lifetime of the whole system. In this paper for finding the best cost route, both the present node energy level and the path for routing is considered at the same time. After all ants achieve the goal to reach the destination, each individual entity ant compares to a path of routing. For optimum route determination, a fitness function is provided where the fitness value can be computed for the path as follows:

$$
f_{(\text {fitness })_{m}^{k}}=\frac{E_{r e s}}{L_{m}^{k}}
$$

where the residual energy level of a sensor node $n_{i}$ for instance is $E_{r e s_{i}} . L_{m}^{k}$ refers to the length of the route for $m^{\text {th }}$ ant and $k^{\text {th }}$ iteration.

The path having the largest value of fitness is viewed as the optimum route.

\section{RESULTS AND DISCUSSION}

The simulation is done using MATLAB where the proposed algorithm is evaluated with the conventional ACO. The comparison of the best cost for the proposed ACO algorithm and for the traditional ACO algorithm, and the energy consumed per transmission of the nodes show the considerable improvement for the proposed method. The simulation results are also compared with the EICAntS algorithm [9] for the best cost route.

The parameters are set for the simulation as follows, no. of ants=40, $\alpha=1, \beta=1, \gamma=1, \rho=0.05$ and considering the initial energy level of each node is 0.5 joule. The mobility parameter utilized here with a speed of $5 \mathrm{~m} / \mathrm{s}$. The data transmitted over a distance $\mathrm{d}=50 \mathrm{~m}$ and received by each node 4000 bits is assumed. With respect to the number of ants, Figure 1 shows the implementation of ACO. From this enactment, we can state that the more prominent the quantity of ants with prior experience and related knowledge better will be the nature of way that the new subterranean insect will pick.

A sensor node basically senses the regional events of the target area and sends information to the sink node. Wireless sensor network deployment is shown in Figure 2 where sensor nodes are dispensed within the area of $100 \mathrm{~m} \times 100 \mathrm{~m}$.

Indeed, the proposed technique is supporting a contrivance permitting the determination of the best route with the end goal to forward the data from the source node to the destination, the base station. The parameters utilized in the choice of paths are refreshed with time and adjusted to the advancement of the system, mobility of the nodes, the quantity of nodes and outstanding vitality of the nodes. 


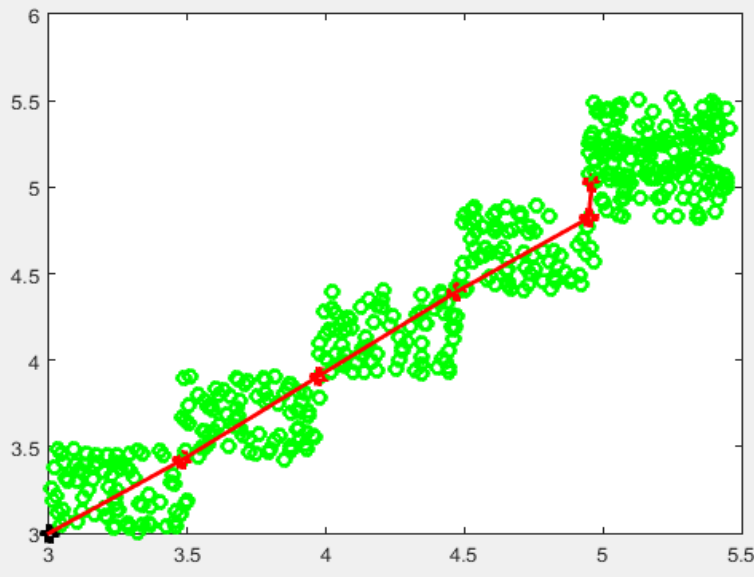

Figure 1. Ant colony optimization implementation

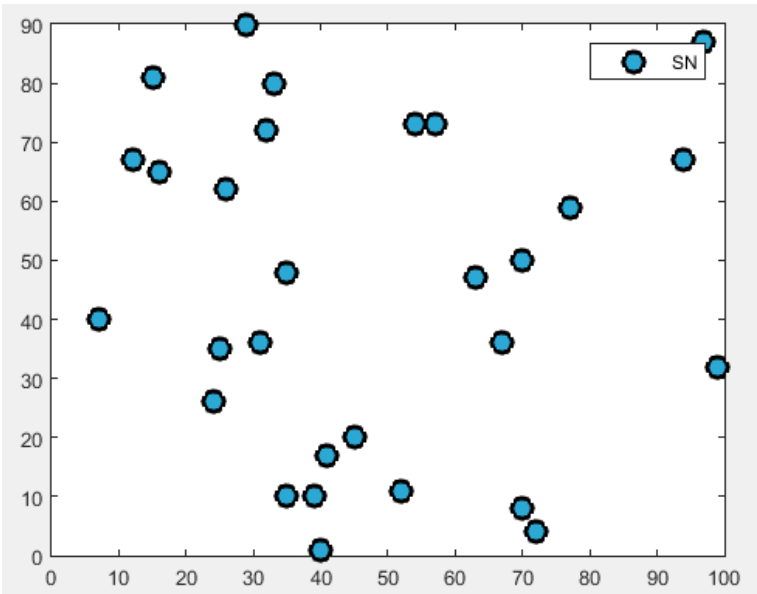

Figure 2. Wireless sensor network deployment

Figure 3 shows the best cost for the proposed ACO algorithm for maximum 300 times iteration which considers the node mobility. Both the node energy and the path for routing have been taken into account. Figure 4 represents the best cost found in traditional ACO algorithm for maximum 300 times iteration, which shows the changes in best cost if the mobility and node energy is not considered.

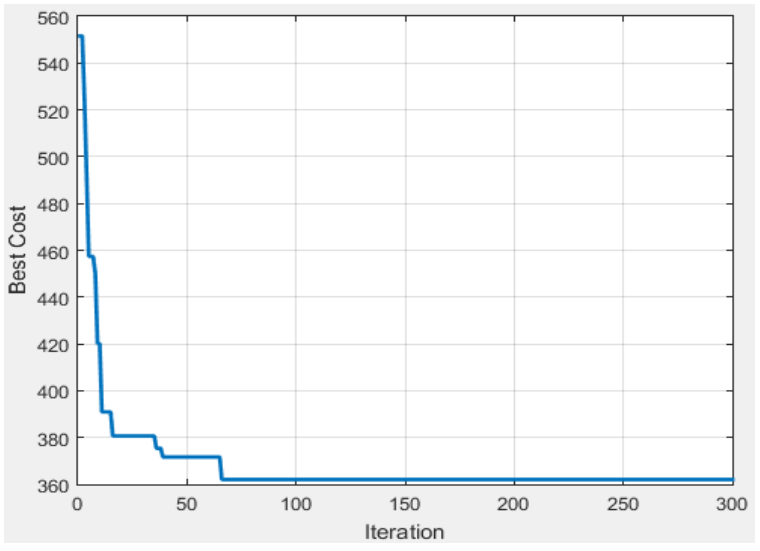

Figure 3. Best cost for the proposed ACO algorithm

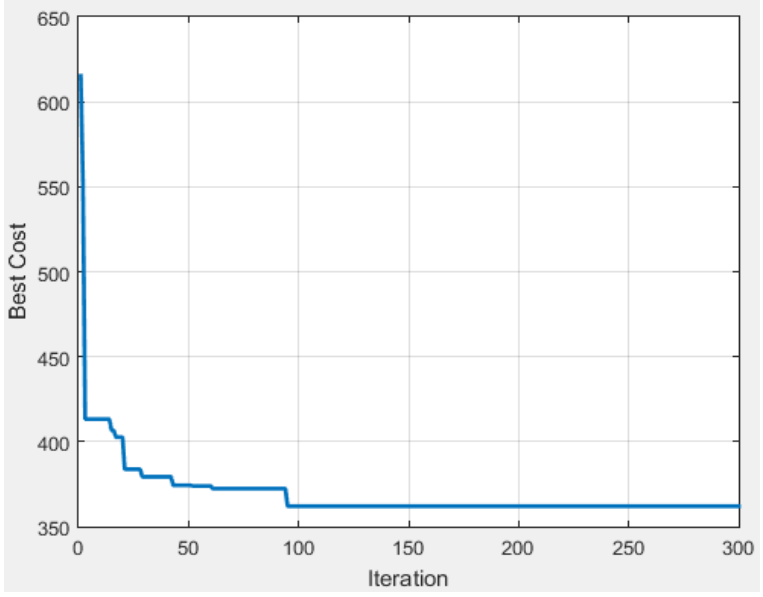

Figure 4. Best cost for the traditional ACO algorithm

Figure 5 shows the comparison of the best cost for the proposed ACO algorithm and for the traditional or conventional ACO algorithm for maximum 300 iterations. The results show that the proposed ACO algorithm can achieve better performance, about $30 \%$ improvement, and faster convergence to determine the best cost route.

Moreover, existing ant based algorithm takes long time for initialization. Here, from the results it is also noticeable that the faster convergence for the initialization delay to find the best cost route for proposed system is achieved compared with the conventional ACO technique.

Incorporating and utilizing the fitness function for the transmission distance of $20 \mathrm{~m}$ and the mobility speed of $2 \mathrm{~m} / \mathrm{sec}$ for the optimal routing path while considering the node energy, the comparative improvement for the best cost for the proposed method with the conventional ACO technique is shown in Figure 6. The comparison of the best cost for the proposed, EICAntS [9] and traditional ACO algorithm is shown in Figure 7. The evaluation results an improvement in convergence to ascertain the best cost path. 


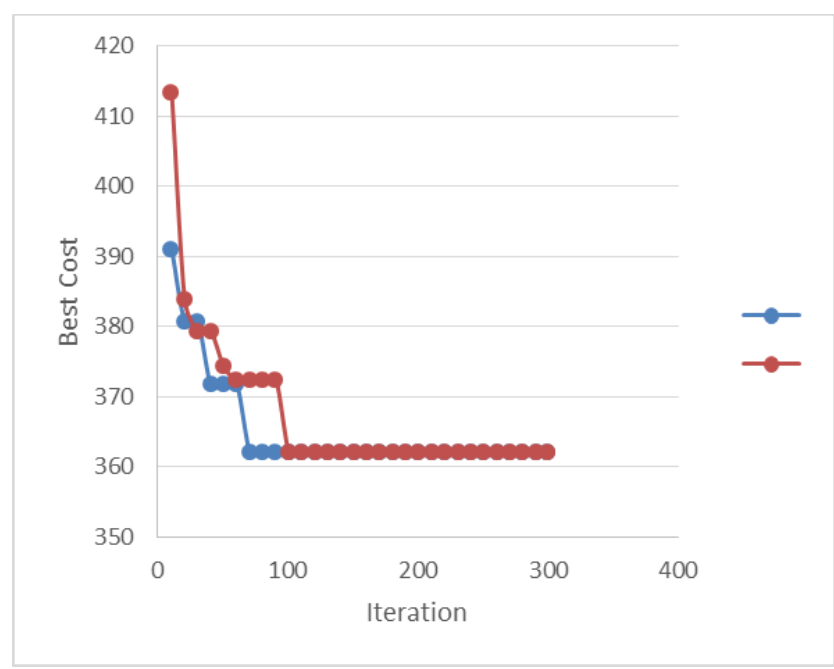

Figure 5. Best cost for the proposed ACO algorithm and traditional ACO algorithm

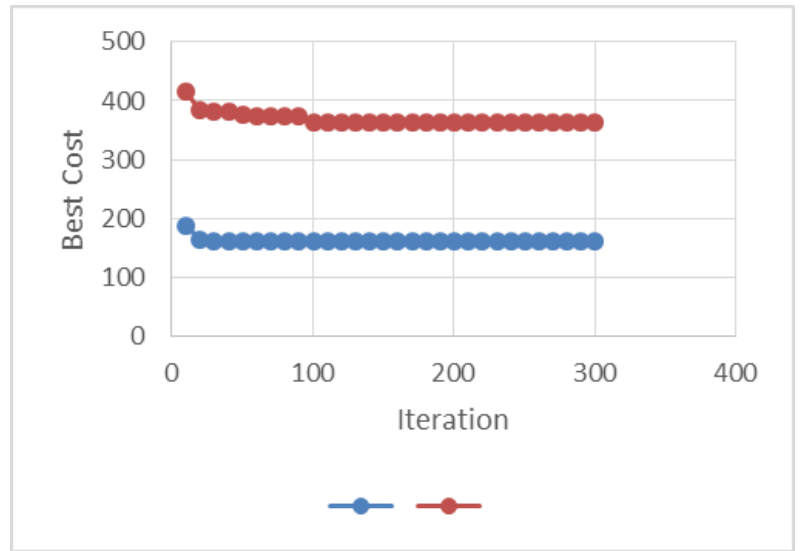

Figure 6. Best cost for the proposed and traditional ACO algorithm using fitness function

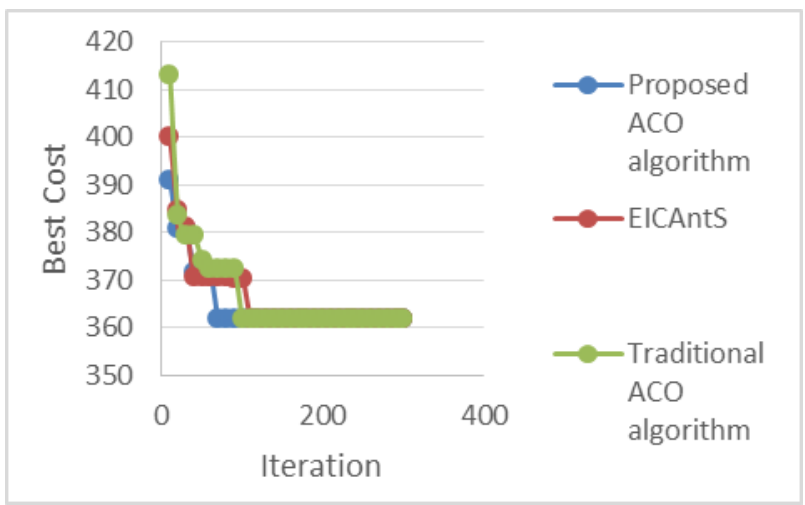

Figure 7. Evaluation of Best cost for the proposed, EICAntS and traditional ACO algorithm (not utilizing fitness function)

The comparison of the best cost for the proposed algorithm and EICAntS algorithm [9] is shown in Figure 8 using fitness function for the transmission distance of $20 \mathrm{~m}$ and the mobility speed of $2 \mathrm{~m} / \mathrm{sec}$, the stated improvement given above for Figure 6. The results show the considerable improvement for the proposed system for varied communication parameters. 


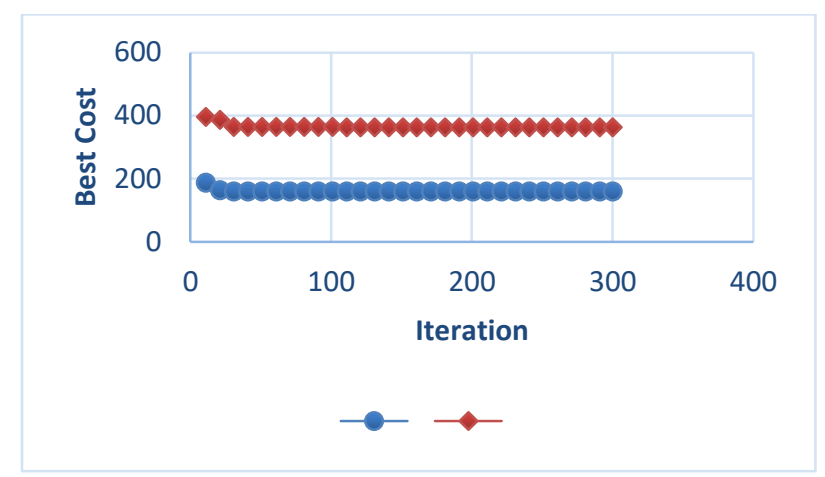

Figure 8. Best cost for the proposed ACO algorithm using fitness function and EICAntS algorithm for varied communication parameters

The comparison of the energy consumed per transmission of the nodes for the proposed ACO algorithm and for the traditional ACO algorithm is shown in Figure 9 which shows the considerable improvement for the proposed method. For distinct search process the aggregate vitality utilization of each node is appeared here per transmission. Plainly for the vast majority of the nodes the proposed calculation has smaller vitality utilization. Contrasted with the conventional calculation the proposed calculation has the undeniable preferred standpoint.

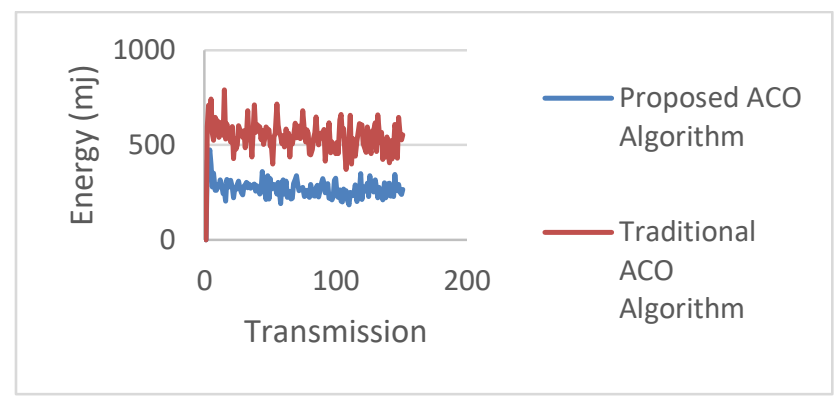

Figure 9. Energy consumption of the node per transmission for the proposed ACO algorithm and traditional ACO algorithm

\section{CONCLUSION}

In this paper, considering key features of the WSNs, the proposed ACO routing algorithm enhances network optimization efficiency. Vital communications parameters and constraints should be considered for the improvement in communications in finding the optimum path for data transmission and to resolve the routing problem whereas taking into account only some parameters is not sufficient to achieve the correspondence quality required by the energy constrained WSNs applications. Thus, here considering the important correspondence parameters, versatility, vitality and route length, reflecting an effective routing calculation in view of ant based system is proposed. The novel technique is utilized for giving a decision for an efficient route and to productive way for transmission for WSNs. An optimization for efficient routing and giving a definitive and reliable route, the energy factor is added and the average mobility, speed of the nodes, to calculate the stability factor is added in the probability formula of an ant colony algorithm which is the distinguishing feature for the new ant algorithm compared to previous work. Performance in terms of best cost and in the energy consumption of the nodes per transmission for the proposed ACO approach is better suited than conventional ACO which has been determined from the comparison. From the simulation results also it is shown that the proposed solution of ACO technique can achieve better enactment, about 30\% improvement compared with the traditional ACO algorithm, and faster convergence to determine the best cost route. Contrasted with the traditional calculation the proposed technique recorded an improvement in the 
energy consumption of the nodes per transmission where for the nodes the proposed calculation has smaller vitality utilization.

Ubiquitous detecting empowered by WSNs innovations cuts across over numerous zones of cutting edge living. The expansion of these devices in a communicating-actuating networked system makes the IoT, wherein sensors and actuators mix flawlessly with nature around us. The issues of the routing of WSNs force nearly similar prerequisites for IoT routing technique. In the future, the large-scale environment will be considered for instance IoT. In addition, the parameters are obtained by means of experiments and how to optimize these parameters and prove in theory is a topic worthy of the continuing research.

\section{REFERENCES}

[1] R. A. L. Rabelo, et al., "An approach based on fuzzy inference system and ant colony optimization for improving the performance of routing protocols in Wireless Sensor Networks," in 2013 IEEE Congress on Evolutionary Computation, pp. 3244-3251, June 2013.

[2] J. Gubbi, R. Buyya, S. Marusic, and M. Palaniswami, "Internet of Things (IoT): a vision, architectural elements, and future directions," Future Generation Computer Systems, vol. 29(7), 1645-1660, 2013.

[3] S. Agnihotri and K. R. Ramkumar, "A survey and comparative analysis of the various routing protocols of Internet of Things," International Journal of Pervasive Computing and Communications, vol. 13(3), 264-281, 2017.

[4] M. R. Dhage and S. Vemuru, "Routing Design Issues in Heterogeneous Wireless Sensor Network," International Journal of Electrical and Computer Engineering (IJECE), vol. 8(2), pp. 1028-1039.

[5] A. Nayyar and R. Singh, "Ant Colony Optimization-computational swarm intelligence technique," In Computing for Sustainable Global Development (INDIACom), 2016 3rd International Conference on IEEE, pp. 1493-1499, March 2016.

[6] T. Gui, C. Ma, F. Wang, and D. E. Wilkins, "Survey on swarm intelligence based routing protocols for Wireless Sensor Networks: an extensive study," in 2016 IEEE International Conference on Industrial Technology, pp. 1944-1949, March 2016.

[7] H. P. Mistry and N. H. Mistry, "A survey: use of ACO on AODV and DSR routing protocols in MANET," In Innovations in Information, Embedded and Communication Systems (ICIIECS), 2015 International Conference on IEEE, pp. 1-6, March 2015.

[8] G. Gajalakshmi and G. U. Srikanth, "A survey on the utilization of Ant Colony Optimization (ACO) algorithm in WSN," In Information Communication and Embedded Systems (ICICES), 2016 International Conference on IEEE, pp. 1-4, February 2016.

[9] O. Deepa and A. Senthilkumar, "Swarm intelligence from natural to artificial systems: Ant colony optimization," Networks (Graph-Hoc), vol. 8(1), pp. 9-17, 2016.

[10] S. Hamrioui and P. Lorenz, "Bio inspired routing algorithm and efficient communications within IoT," IEEE Network, vol. 31(5), pp. 74-79, 2017.

[11] M. Umadevi and M. Devapriya, "An enhanced ant colony based approach to optimize the usage of critical node in Wireless Sensor Networks," Procedia Computer Science, vol. 47, pp. 452-459, 2015.

[12] J. Kaur and G. Kaur, "An amended ant colony optimization based approach for optimal route path discovery in wireless sensor network," in 2017 IEEE International Conference on Smart Technologies and Management for Computing, Communication, Controls, Energy and Materials (ICSTM), pp. 353-357, August 2017.

[13] X. Liu, S. Li, and M. Wang, "An ant colony based routing algorithm for Wireless Sensor Network," International Journal of Future Generation Communication and Networking, vol. 9(6), pp. 75-86, 2016.

[14] R. A. Mahale and S. D. Chavan, "Throughput aware ACO based routing protocol for wireless sensor network," in 2014 IEEE Global Conference on Wireless Computing and Networking (GCWCN), pp. 234-238, December 2014.

[15] T. Camilo, et al., "An Energy-Efficient Ant-Based Routing Algorithm for Wireless Sensor Networks," In Internationnal Workshop on Ant Colony Optimization and Swarm Intelligence, Springer, Berlin, Heidelberg, pp.49-59, 2006.

[16] A. Sharmin, F. Anwar and S. M. A. Motakabber, "A Noble Approach of ACO Algorithm for WSN," 20187 th International Conference on Computer and Communication Engineering (ICCCE), Kuala Lumpur, 2018, pp. $152-156$

[17] W. B. Heinzelman, A. P. Chandrakasan and H. Balakrishnan, "An application-specific protocol architecture for wireless microsensor networks," in IEEE Transactions on Wireless Communications, vol. 1, no. 4, pp. 660-670, Oct. 2002.

[18] F. Anwar, M. Masud, B.U. Islam, R.F. Olanrewaju, "Radio Access Technology (RAT) Selection Mechanism using TOPSIS Method in Heterogeneous Wireless Networks (HWN)," Indonesian Journal of Electrical Engineering and Computer Science (IJEECS), 2018, 12(2), pp. 852-864. 


\section{BIOGRAPHIES OF AUTHORS}

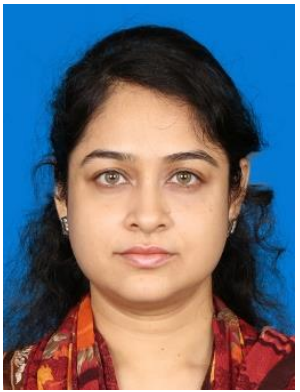

Afsah Sharmin received an M.Sc. in Telecommunications Engineering and Telecommunication Networks from the University of Technology Sydney (UTS), Australia in 2009 and a B.Sc. in Computer Engineering from American International University-Bangladesh (AIUB), Bangladesh in 2004. She is currently a Master by Research student at the International Islamic University Malaysia (IIUM) and is conducting research in Computer and Information Engineering. Her research interests are in wireless sensor networks, bio inspired WSN routing algorithm, energy efficient communication and IoT. She had been a faculty member of American International University-Bangladesh in the Department of Computer Science and Engineering.

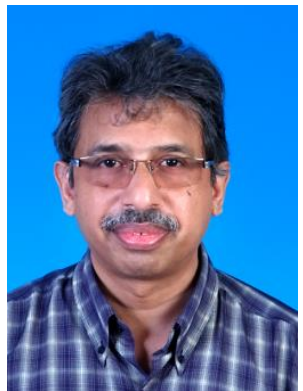

Dr. Farhat Anwar received a $\mathrm{PhD}$ degree in Electronic and Electrical Engineering from the University of Strathclyde UK in 1996 and his MSc from the University of Dhaka, Bangladesh. His research interest includes QoS in IP networks, routing in Ah-hoc and sensor networks, computer simulation and performance analysis, computer security, and biometrics. He has published extensively with over 140 technical papers in international journals and conferences and 30 book chapters. He has been with IIUM since 1999 and currently working as a Professor and coordinator of computer networking research group in the department of Electrical and Computer Engineering.

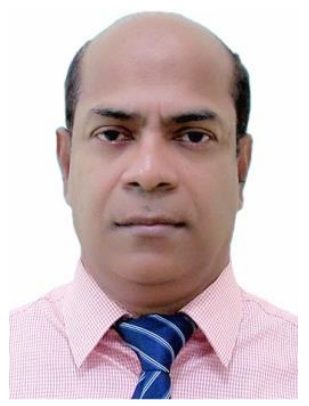

S. M. A. Motakabber was born in a highly educated Muslim family on 31st May, 1966 at Naogaon District of Bangladesh. He received the B.Sc. and M.Sc. Degrees in Applied Physics and Electronics from the University of Rajshahi, in 1986 and 1987, respectively, and the Ph.D. degree in Electrical, Electronic and Systems Engineering from the National University of Malaysia, in 2011. From 1993 to 2011, he was an Associate Professor in the Department of Applied Physics and Electronic Engineering at the University of Rajshahi, Bangladesh. He is now Assistant Professor in the Department of Electrical and Computer Engineering at the International Islamic University of Malaysia. Dr. Motakabber is a chartered engineer UK (CEng). He is a senior member of the Institute of Electrical and Electronics Engineers (IEEE), Institution of Engineering and Technology (IET), a life member of the Bangladesh Association for Advancement of Science (BAAS), and the Bangladesh Electronics Society (BEC). His research interests are Analog \& Digital Electronic System Design, Wireless Sensor Networking, Medical \& Industrial Instrumentation, VLSI Design, RFID, Robotics, Automated Control System, Embedded System Design and IoT. He has published about 40 research articles in peer-reviewed journals, 60 conference proceedings, one textbook for under grate students and two research books. 